Original Research Paper

\title{
Model of Adsorption on Epitaxial Single-Layer Graphene
}

\author{
${ }^{1,2}$ Davydov Sergei and ${ }^{1,2}$ Lebedev Alexander \\ ${ }^{1}$ Ioffe Physical Technical Institute, 194021, St. Petersburg, Russia \\ ${ }^{2}$ National Research University of Information Technologies, Mechanics and Optics, 197101, St. Petersburg, Russia
}

Article history

Received: 22-09-2014

Revised: 03-10-2014

Accepted: 04-07-2015

Corresponding Author:

Davydov Sergei

Ioffe Physical Technical

Institute, 194021, St.

Petersburg, Russia

Email: Sergei_Davydov@mail.ru

\begin{abstract}
Model of adsorption on graphene is built by two steps: For the beginning density of states for the graphene monolayer on solid substrate is calculated, then adsorbed atom is placed on the such constructed epitaxial graphene. Both metallic and semiconducting substrates are considered. Charge transfer between adatom and epitaxial graphene is estimated. Effects of graphene layer and substrate on the electron state of adatom are analyzed.
\end{abstract}

Keywords: 3C-SiC, Epilayer, Substrates, Graphene

\section{Introduction}

The problem on adsorption on the single-layer Epitaxial Graphene (EG) is of interest firstly since EG is the basic element of the planar device structures (Castro Neto et al., 2008; Haas et al., 2008; Wu et al., 2010; Cooper et al., 2011).

The second fundamental aspect of this problem is connected with the two questions: (I) how and in what extent electronic state of the adsorbed particle is connected with the graphene layer from the one hand and with the substrate from the other hand; (II) how adatom effects graphene electronic spectrum.

Adsorption properties of the single-layer free-Standing Graphene (SLG) where studied earlier in the scope of the so-called M-model (Davydov and Sabirova, 2011; Davydov, 2011a). Here we also will use the model approach to the EG adsorption problem. We will construct corresponding model step-by-step, beginning with the EG model and then transfer to the adsorption problem.

\section{General Relations}

The problem on electron states of EG was considered by us (see, for example, Davydov (2013) and references therein). For the corresponding Green function $G_{e g}(\omega$, $k$ ), where $\mathrm{k}=\left(k_{x}, k_{y}\right)$ is the two-dimensional SLG wave vector we have obtained:

$$
\begin{aligned}
& G_{e g}^{-1}(\omega, \mathrm{k})=\Omega-\Lambda_{e g}(\omega)+i \Gamma_{e g}(\omega) \mp t f(\mathrm{k}), \\
& f^{2}(\mathrm{k})=3+2 \cos \left(k_{x} a \sqrt{3}\right)+4 \cos \left(k_{x} a \sqrt{3} / 2\right) \cos \left(3 k_{y} a / 2\right)
\end{aligned}
$$

Here $\Omega=\omega-\varepsilon_{c}$, where $\omega$ is the energy variable, $\varepsilon_{c}$ is the Dirac point energy for the free-standing SLG, corresponding to the $\left|p_{z}\right\rangle$ state of the carbon atom, the carbon quasilevel half-width function
$\Gamma_{e g}(\omega)=\pi V_{s g}^{2} \rho_{s u b}(\omega)$, where $V_{s g}$ is the substrate-graphene hopping energy, $\rho_{s u b}(\omega)$ is the substrate Density of States (DOS), $t$ is the hopping energy for the $\left|p_{z}\right\rangle$ states of the nearest-neighbor carbon atoms separated by the distance $a$, the carbon quasilevel shift function:

$$
\Lambda_{e g}(\omega)=\frac{1}{\pi} P \int_{-\infty}^{\infty} \frac{\Gamma_{e g}\left(\omega^{\prime}\right) d \omega^{\prime}}{\omega-\omega^{\prime}} d \omega^{\prime}
$$

where, $P$ is the principal value symbol, the upper sign in (1) is related to the conduction $\pi^{*}$ band $\left(\bar{\Omega} \equiv \Omega-\Lambda_{e g}(\omega)>0\right)$, the lower sign is related to the valence $\pi$ band $(\bar{\Omega}<0)$. Then the low-energy free-standing SLG spectrum approximation (Castro Neto et al., 2008) was used:

$$
\varepsilon_{ \pm}(\mathrm{q})=\varepsilon_{c} \pm \frac{3}{2} t a|\mathrm{q}|
$$

where, $q=K-k, \mathrm{~K}=a^{-1}(2 \pi / 3 \sqrt{3}, 2 \pi / 3)$ is the Dirac point wave vector. Then:

$G_{e g}^{-1}(\bar{\Omega}, q)=\bar{\Omega}+i \Gamma_{e g}(\omega) \mp \frac{3 t}{2}(a q)$

where, $q=|q|$. DOS for EG has the form (Davydov, 2011a):

$$
\begin{aligned}
& \rho_{e g}(\omega)=\frac{1}{\pi \xi^{2}}\left[\Gamma_{e g}(\omega) \ln \frac{(\bar{\Omega} \mp \xi)^{2}+\Gamma_{e g}^{2}(\omega)}{\bar{\Omega}^{2}+\Gamma_{e g}^{2}(\omega)}+2 \bar{\Omega}\right. \\
& \left.\left(\arctan \frac{\bar{\Omega}}{\Gamma_{e g}(\omega)}-\arctan \frac{\bar{\Omega} \mp \xi}{\Gamma_{e g}(\omega)}\right)\right]
\end{aligned}
$$


where, $\xi=3 \operatorname{taq}_{B} / 2$ is the cut-off energy with the cut-off wave vector.

Now let us turn to the adsorption on EG. From the general point of view the adatom's Green function $G_{a}(\omega)$ for this case can be written as:

$$
G_{a}^{-1}(\omega)=\omega-\varepsilon_{a}-\Lambda_{a}(\omega)+i \Gamma_{a}(\omega)
$$

Here $\varepsilon_{a}$ is the energy of the adatom's quasilevel, which is participated in the charge-transfer process, the adatom's quasilevel half-width function $\Gamma_{a}(\omega)=\pi V_{a / e g}^{2} \rho_{e g}(\omega)$, where $V_{a / e g}$ is the adatom-EG hopping energy, the adatom's quasilevel shift function:

$$
\Lambda_{a}(\omega)=\frac{1}{\pi} P \int_{-\infty}^{\infty} \frac{\Gamma_{a}\left(\omega^{\prime}\right) d \omega^{\prime}}{\omega-\omega^{\prime}}
$$

The adatom's DOS $\rho_{a}(\omega)$, corresponding to the Green function (6), is:

$$
\rho_{a}(\omega)=\frac{1}{\pi} \frac{\Gamma_{a}(\omega)}{\left[\omega-\varepsilon_{a}-\Lambda_{a}(\omega)\right]^{2}+\Gamma_{a}^{2}(\omega)}
$$

and the adatom's occupation number $n_{a}$ for the zero temperature is:

$$
n_{a}=\int_{-\infty}^{\varepsilon_{F}} \rho_{a}(\omega) d \omega
$$

where, $\varepsilon_{F}$ is the Fermi level energy.

\section{Metallic Substrate}

We take the metallic substrate DOS equal to constant value $\rho_{m}$ as for the Anderson model within the scope of the wide-band approximation (Anderson, 1961). Then $\Gamma_{e g}(\omega)=\Gamma_{m}=\pi V_{g / m}^{2} \rho_{m}=$ const and $\Lambda_{e g}(\omega) \equiv \Lambda_{m}=0$ ( $V_{g / m}$ is the graphene-metal hopping energy). In this case instead of Equation 5 we get:

$$
\rho_{e g}^{m}(\omega)=\frac{1}{\pi \xi^{2}}\left[\begin{array}{l}
\Gamma_{m} \ln \frac{(\Omega \mp \xi)^{2}+\Gamma_{m}^{2}}{\Omega^{2}+\Gamma_{m}^{2}} \\
+2 \Omega\left(\arctan \frac{\Omega}{\Gamma_{m}}-\arctan \frac{\Omega \mp \xi}{\Gamma_{m}}\right)
\end{array}\right]
$$

Estimations made in (Davydov, 2011b) shown that Equation 10 can be approximated by the expression:

$$
\rho_{e g}^{m}(\omega) \approx \frac{1}{\pi} \frac{\Gamma_{m}}{\left(\omega \mp \omega_{\max }\right)^{2}+\Gamma_{m}^{2}}
$$

where, we put $\varepsilon_{c}=0$, the upper sing is referred to $\omega>0$, the lower sign is referred to $\omega<0$ and:

$$
\begin{aligned}
& \omega_{\max }=\frac{\xi}{2}+\delta \\
& \delta \approx \frac{\Gamma_{m}^{2}}{4 \xi}\left(\frac{4 \Gamma_{m}^{2}+\xi^{2}}{2 \Gamma_{m} \xi} \arctan \left(\frac{\xi}{2 \Gamma_{m}}\right)-1\right)
\end{aligned}
$$

It is easy to see that for $\beta_{m}=\left(\xi / \Gamma_{\mathrm{m}}\right)<<1$ we arrive at $\omega_{\max } \approx \xi / 2$. In the cast of $\beta_{m}>>1$ we obtain $\omega_{\max } \approx$ $(\xi / 2)+\left(\pi \Gamma_{\mathrm{m}} / 16\right)$.

Note that the parameter $\xi$ defines the half-width of the linear $\rho_{g}(\omega)$ dependence energy interval centered at the Dirac point $\omega_{D}=\varepsilon_{c}$. Actually, in the low-energy approximation we have (see, for example, (Davydov and Sabirova, 2011; Davydov, 2013):

$\rho_{g}(\omega)=2 \frac{\left|\omega-\varepsilon_{c}\right|}{\xi^{2}}$

Then we estimate $\xi \leq t \sim 3 \mathrm{eV}$. Since $\beta_{m} \sim t / \Gamma_{m}$, then the limit $\beta_{m}<<1$ can be interpreted as the strong-coupling graphene-substrate regime (in compare with the interatomic graphene coupling); in the opposite limit $\beta_{m}>>1$ the weak-coupling regime is realized.

Since $\Gamma_{a}(\omega)=\pi V_{a / e g}^{2} \rho_{e g}^{m}(\omega)$, taking $\rho_{e g}(\omega)$ from (11) and using (7), we find:

$$
\Lambda_{a}(\omega)=\frac{V_{a / e g}^{2} \Gamma_{m}}{\left(\omega_{\max }-|\omega|\right)^{2}} \ln \left|\frac{\omega-\omega_{\max }}{\omega+\omega_{\max }}\right|
$$

It is easy to demonstrate that in the case of $|\omega| \gg>\omega_{\max }$ shift-function $\Lambda_{a}(\omega) \approx-2 V_{a / e g}^{2} \Gamma_{m} \omega_{\max } / \omega^{3}$, while in the opposite limit $\quad|\omega|<<\omega_{\max } \quad$ we obtain $\Lambda_{a}(\omega) \approx-2 V_{a / e g}^{2} \Gamma_{m} \omega / \omega_{\max }^{3}$.

Now we introduce dimensionless values: $x=\omega / \Gamma_{m}$, $x_{\max }=\omega_{\max } / \Gamma_{m}, e_{a}=\varepsilon_{a} / \Gamma_{m}, \quad v=V_{a / e g} / \Gamma_{m}$. The reduced shift functions $\lambda_{a}=\Lambda_{a} / \Gamma_{m}$, half-width function $\gamma_{a}=\Gamma_{a} / \Gamma_{m}$, DOS of EG $\bar{\rho}_{e g}^{m}=\rho_{e g}^{m} \Gamma_{m}$ and adatom's DOS $\bar{\rho}_{a}=\rho_{a} \Gamma_{m}$ are equal correspondingly:

$$
\begin{aligned}
& \lambda_{a}(x)=\frac{v^{2}}{\left(x_{\max }-|x|\right)^{2}} \ln \left|\frac{x-x_{\max }}{x+x_{n a x}}\right|, \quad \gamma_{a}(x)=\pi v^{2} \bar{\rho}_{e g}^{m}(x), \\
& \bar{\rho}_{e g}^{m}(x)=\frac{1}{\pi} \frac{1}{\left(x-x_{\max }\right)^{2}+1}, \quad \bar{\rho}_{a}(x)=\frac{1}{\pi} \frac{\gamma_{a}(x)}{\left[x-e_{a}-\lambda_{a}(x)\right]^{2}+\gamma_{a}^{2}(x)}
\end{aligned}
$$

For the strong-coupling regime $\left(\beta_{m}<<1\right)$ we find $x_{m}$ $\approx 0$, for a weak-coupling regime $\left(\beta_{m}>>1\right)$ we have $x_{m}$ $\approx \pi / 16$, which also can be considered as a small value. Thus, for the illustrative purposes only we can restrict our consideration by the case $x_{\max }=0$, which gives $\lambda(x)=0$. 


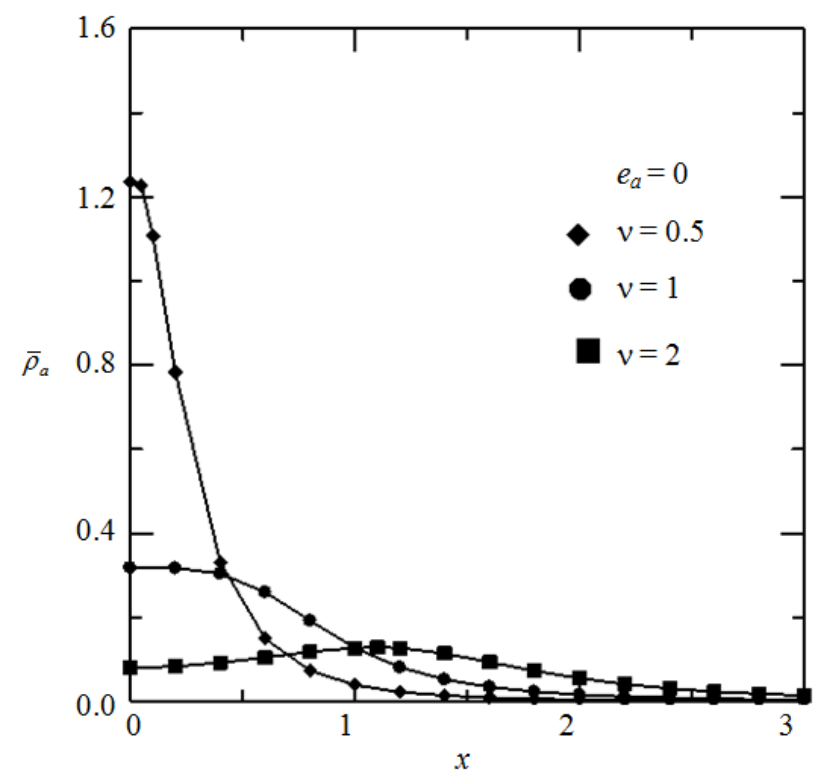

(a)



(b)

Fig. 1. (a) Metallic substrate: Adatom's reduced DOS $\bar{\rho}_{a}(x)$ dependence on the dimensionless energy $x$ for $x_{m}=0$ and $v=0.5$ (rhombs), 1 (circles) и 2 (squares) (a) $\varepsilon_{a}=0$ (only right hand-side of the symmetric curve is shown), (b) Metallic substrate: Adatom's reduced DOS $\bar{\rho}_{a}(x)$ dependence on the dimensionless energy $x$ for $x_{m}=0$ and $v=0.5$ (rhombs), 1 (circles) и 2 (squares). (b) $\varepsilon_{a}=1$

Figure 1 demonstrates the typical dependencies of the adatom's DOS $\bar{\rho}_{a}$ on the dimensionless energy $x$ for the three values of parameter $v$. The obtained differences for the cases $v=0.5,1$ and 2 can be understood as follows. Firstly, the maximal value of the adatom's DOS is $\bar{\rho}_{a \max } \propto v^{-2}$ (рис. 1). Secondly, for $\varepsilon_{a}=0$ maximums of $\bar{\rho}_{a}(0)$ appear at $v \leq 1$, minimums appear at $v>1$ (Fig. 1a). Thirdly, $\bar{\rho}_{a}(x)$ maximums shift to the greater $x$ (for $\varepsilon_{a}$ $>0$ ) with the $v$ increase (Fig. 1b) is connected with the character of positive root $x^{*}$ of the equation $d \bar{\rho}_{a}(x) / d x=0$, which can be estimated for $\kappa<1$ as:

$x^{*} \approx e_{a}\left(1+\frac{v^{4}}{\left(e_{a}^{2}+1\right)^{3}}\right)$

Underline, that the inequality $v=\left(V_{\text {aleg }} / \Gamma_{m}\right)<1$ corresponds to the excess of the graphene-substrate coupling compared to the adatom-graphene coupling, the opposite inequality $v>1$ corresponds to the excess of adatom-graphene coupling.

To calculate the adatom's occupation number $n_{a}$ we make some extra simplification taking $\gamma_{a}^{*}=\gamma_{a}\left(x^{*}\right)$ instead of $\gamma a(x)$. Then for $k<1$ we find:

$n_{a} \approx \frac{1}{\pi} \operatorname{arcctg} \frac{e_{a}-e_{F}}{\gamma_{a}^{*}}$ where, $e_{F}=\varepsilon_{F} / \Gamma_{m}$. Increase of $v$ leads to decrease of $\gamma^{*}{ }_{a}$ and $n_{a}$ increases for $e_{a}<e_{F}$ and decreases for $e_{a}>e_{F}$. Thus, the increase of the adatom-EG coupling compared to graphene-metal charge transfer decreases for $e_{a}<e_{F}$ and increases for $e_{a}>e_{F}$.

\section{Semiconducting Substrate}

In this case we take DOS $\rho_{s c}(\omega)$ in the form (Davydov, 2014a):

$\rho_{s c}(\omega)=A \cdot \begin{cases}\sqrt{\omega-E_{g} / 2}, & \omega>E_{g} / 2, \\ \sqrt{-\omega-E_{g} / 2}, & \omega<-E_{g} / 2, \\ 0, & |\omega| \leq E_{g} / 2\end{cases}$

Here $A$ is the coefficient, $E_{g}$ is the energy gap width, zero of energy coincides with the gap center.

As it was shown in (Davydov, 2014b), EG DOS $\rho_{e g}^{s c}(\omega)$ is given by Equation 5 provided $\Gamma_{e g}(\omega)$ is changed by $\Gamma_{s c}(\omega)$ and $\Lambda_{e g}(\omega)$ is changed by $\Lambda_{s c}(\omega)$, where.

where, $F_{ \pm}(\omega)=\pi \sqrt{ \pm \omega+E_{g} / 2}$.

$\Gamma_{s c}(\omega)=\pi V_{g / s c}^{2} \rho_{s c}(\omega), V_{g / s c}$ is the graphene-substrate hopping energy and: 
$\Lambda_{s c}(\omega)=A V_{g / s c}^{2} \cdot \begin{cases}F_{-}(\omega), & \omega<-E_{g} / 2, \\ F_{-}(\omega)-F_{+}(\omega), & |\omega| \leq E_{g} / 2, \\ -F_{+}(\omega), & \omega>E_{g} / 2,\end{cases}$

Let us consider function $\rho_{e g}^{s c}(\omega)$ in the energy domain $|\omega|<E_{g} / 2$. Within this domain function $\Gamma_{s c}(\omega)$ is equal identically to zero, so that Equation 5 transforms to:

$\rho_{e g}^{s c}(\omega)=\frac{2 \bar{\Omega}}{\pi \xi^{2}}\left(\operatorname{arctg} \frac{\bar{\Omega}}{s}-\operatorname{arctg} \frac{\bar{\Omega} \mp \xi}{s}\right)$

where, $\quad \bar{\Omega}=\omega-\varepsilon_{c}-\Lambda_{s c}(\omega), \quad s=0^{+}$. As it was demonstrated in (Davydov, 2011a), the value of $\rho_{e g}^{s c}(\omega)$ becomes zero for $|\bar{\Omega}|>\xi$. If this inequality does not satisfied, EG DOS in the energy interval has the form:

$\rho_{e g}^{s c}(\omega)=\frac{2|\bar{\Omega}|}{\xi^{2}}$

Which in the case of $\Lambda_{s c}(\omega)=0$ coincides with the free standing SLG DOS $\rho_{g}(\omega)$, given by Equation 13 .

Within the energy region $|\omega|<E_{g} / 2$ adatom's quasilevel shift function:

$$
\Lambda_{s c}(\omega)=\pi A V_{g / s c}^{2}\left[\sqrt{-\omega+E_{g} / 2}-\sqrt{\omega+E_{g} / 2}\right]
$$

Has minimal value equal to $\Lambda_{s c}(0)=0$ and maximal value equal to $\Lambda_{\max }=\Lambda_{s c}\left( \pm E_{g} / 2\right)=\pi A V_{g / s c}^{2} \sqrt{E_{g}}$. In an analogy with the metallic substrate, let us introduce ratio $\beta_{s c}=\xi / \Lambda_{\max }$ and will address the case $\beta_{s c}<<1$ as the graphene-substrate strong-coupling regime, the opposite case when $\beta_{s c}>1$ will be addressed as the weakcoupling regime. Here, as in sec. $2, \beta_{s c} \sim t / \Lambda_{\max }$. In the first case $\left(\beta_{s c}<<1\right)$ the substrate energy gap $E_{g}$ crucially manifests itself in the resulting spectrum of EG. In the second case $\left(\beta_{s c}>1\right)$ we obtain the so called quasi-free-standing EG (Davydov, 2014a), having gapless DOS but with the shifted Dirac point from the energy $\varepsilon_{D}=\varepsilon_{c}$ to $\varepsilon_{D}^{\prime}=\varepsilon_{c}+\Lambda_{s c}\left(\varepsilon_{D}^{\prime}\right)$.

Let for simplicity $\varepsilon_{c}=0$, i.e., $\varepsilon_{c}$ coincides with the center of the substrate band gap $E_{g}$. This excludes the charge transfer between undoped graphene and the intrinsic semiconducting substrate. The gapless EG spectrum condition is the fulfillment of the inequality $\Lambda_{\max }<\left(\xi-E_{g} / 2\right)$. Since $\xi \sim t \approx 3 \mathrm{eV}$ and $E_{g} \backslash 2=1.5 \mathrm{eV}$ for the $6 \mathrm{H}-\mathrm{SiC}$ polytype, the above inequality becomes $\Lambda_{\text {max }}<1.5 \mathrm{eV}$. Estimations of the (Davydov, 2014b) give $A \approx 0.2 \mathrm{eV}^{-3.2}$ and $\Lambda_{\max } \approx 1 \mathrm{eV}^{-1} \cdot V_{g / s c}^{2}$.
DOS on the atom adsorbed on EG is given by Equation 8, where $\Gamma_{a}(\omega)=\pi V_{a / \text { leg }}^{2} \rho_{e g}^{s c}(\omega)$ and:

$$
\Lambda_{a}(\omega)=V_{a / e g}^{2} \int_{-\infty}^{\infty} \frac{\rho_{e g}^{s c}\left(\omega^{\prime}\right) d \omega^{\prime}}{\omega-\omega^{\prime}}
$$

Let us introduce dimensionless values: $y=\omega / E_{g}, e_{c}=$ $2 \varepsilon_{c} / E_{g}, \quad a=A\left(E_{g} / 2\right)^{3 / 2}, \quad \xi=2 \xi / E_{g}, \quad w=2 V_{g / s d} / E_{g}$, $\bar{\rho}_{e g}^{s c}(y)=\rho_{e g}^{s c} \cdot\left(E_{g} / 2\right)$. Dependences of the reduced EG DOS $\bar{\rho}_{e g}^{s c}$ on the dimensionless energy $y$ are shown in Fig. 2 for the energy domain corresponding to the substrate valence band. Note that the function $\bar{\rho}_{e g}^{s c}(y)$ for $e_{c}=0$ is symmetric relative to $y=0$; function $\bar{\rho}_{e g}^{s c}(y)$ for $y<-1$ and $e_{c}=C$, where $C$ is some number, does not change provided $y>1$ and $e_{c}=-C$. Figure 3 demonstrates $\bar{\rho}_{e g}^{s c}(y)$ dependences for the energy domain corresponding to the substrate energy gap $E_{g}$. Note that in Fig. 3a the $\bar{\rho}_{e g}^{s c}(y)$ dependence for $e_{c}=1.5$ does not shown since it can be obtained from the $\bar{\rho}_{e g}^{s c}(y)$ для $e_{c}=-1.5$ dependence be the mirror reflection relative to $y=0$; Fig. $3 \mathrm{~b}$ demonstrates only one-half of the symmetric $\bar{\rho}_{e g}^{s c}(y)$ function for $y<0$.

It follows from Fig. 2, that for $w=1, e_{c}=0,1.5$ (Fig. 2a) and $w=2, e_{c},=0$ (Fig. 2b) $\rho_{e g}^{s c}(y)$ function can be approximated by the constant $\rho_{v a l}$ for the energies corresponding to the valence and conduction bands. Then, taking into account Equation 21, we find for the shift function $\Lambda_{a}(\omega)$ following expression.

$$
\begin{aligned}
& \Lambda_{a}(\omega)=\rho_{\text {val }} V_{\text {aleg }}^{2} \ln \left|\frac{\omega-E_{g} / 2}{\omega+E_{g} / 2}\right|+I, \\
& I=\frac{2 V_{\text {aleg }}^{2}}{\xi^{2}} \int_{\omega_{-}}^{\omega_{+}} \frac{\left|\omega^{\prime}-\varepsilon_{c}-\Lambda_{s c}\left(\omega^{\prime}\right)\right|}{\omega-\omega^{\prime}} d \omega^{\prime},
\end{aligned}
$$

where, $\omega_{-(+)}$is the bottom (top) of the EG of the EG sub and within the substrate gap energy domain. Taking into account results obtained here (Fig. 3) and in (Davydov, 2014a) (Fig. 1 in (Davydov, 2014b)), replace function $\Lambda_{\mathrm{sc}}(\omega)$ given by Equation 22 by its approximate value $\Lambda_{s c}(\omega) \approx-A V_{g / s c}^{2} \sqrt{2 / E_{g}} \omega$. Then we can express integral $I$ in the form:

$I=\frac{2 V_{a / \text { eg }}^{2}}{\xi^{2}} \int_{\omega_{-}}^{\omega_{+}} \frac{\left|\alpha \omega^{\prime}-\varepsilon_{c}\right|}{\omega-\omega^{\prime}} d \omega^{\prime}$

where, $\alpha=1+A V_{g / s c}^{2} \sqrt{2 / E_{g}}$. Thus:

$$
I \approx \frac{2 V_{a / e g}^{2}}{\xi^{2}}\left(\begin{array}{l}
2 \varepsilon_{c}-\alpha\left(\omega_{+}+\omega_{-}\right)+\left(\varepsilon_{c}-\alpha \omega\right) \\
\ln \left|\frac{\left(\omega-\omega_{+}\right)\left(\omega-\omega_{-}\right)}{\left(\omega-\varepsilon_{c} / \alpha\right)^{2}}\right|
\end{array}\right)
$$




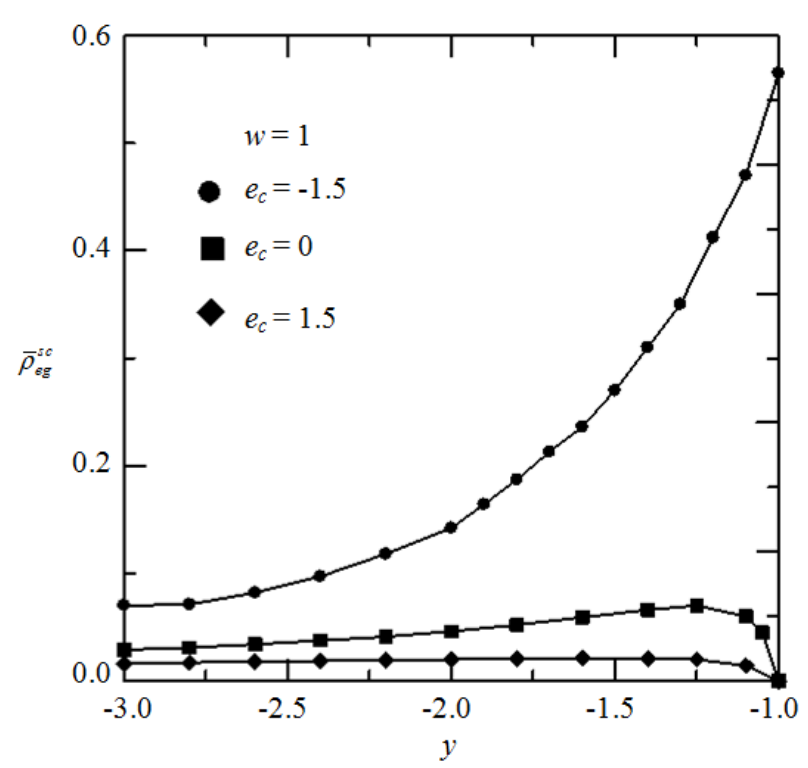

(a)

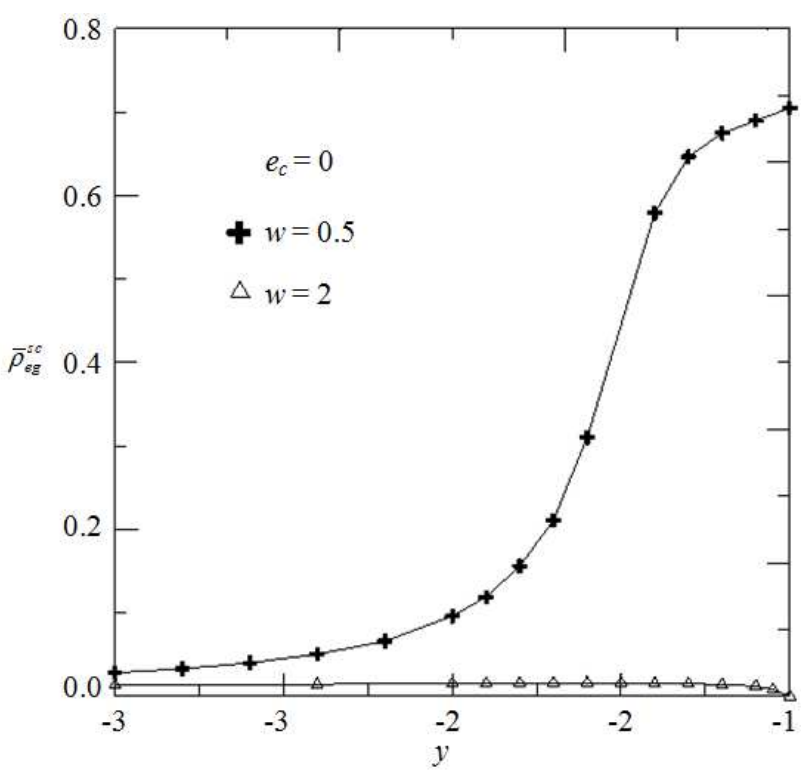

(b)

Fig. 2. (a) Semiconducting substrate: EG reduced DOS dependence on the dimensionless energy for $\approx 0.37,=2$ and (a), $=\square 1.5$ (circles), 0 (squares), 1.5 (rhombs), (b) Semiconducting substrate: EG reduced DOS dependence on the dimensionless energy for $\approx 0.37,=2$ and $(b)=0$, (crosses), 2 (triangles). Only the energy domain corresponding to the substrate valence band is shown

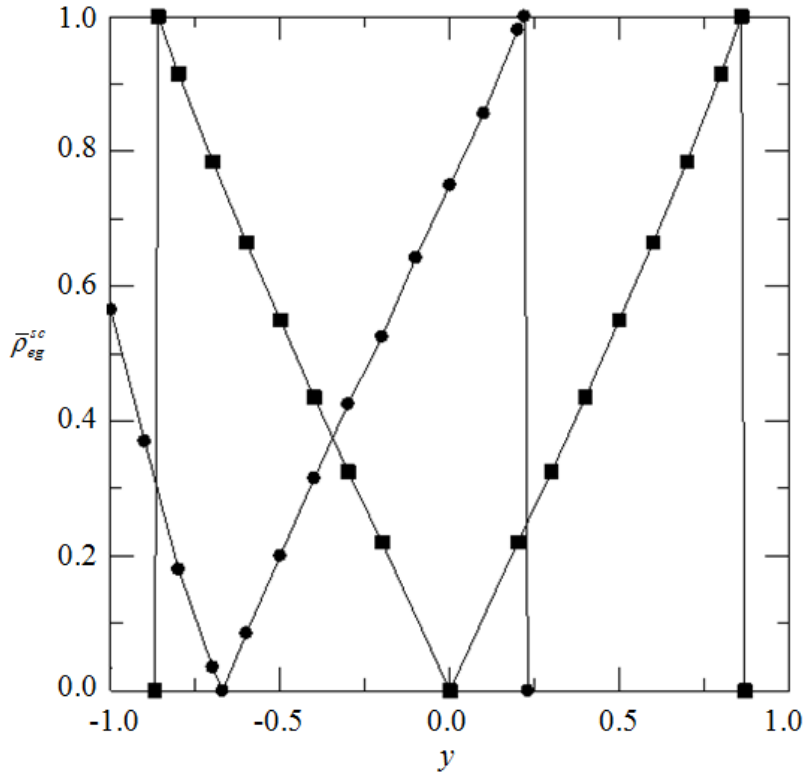

(a)

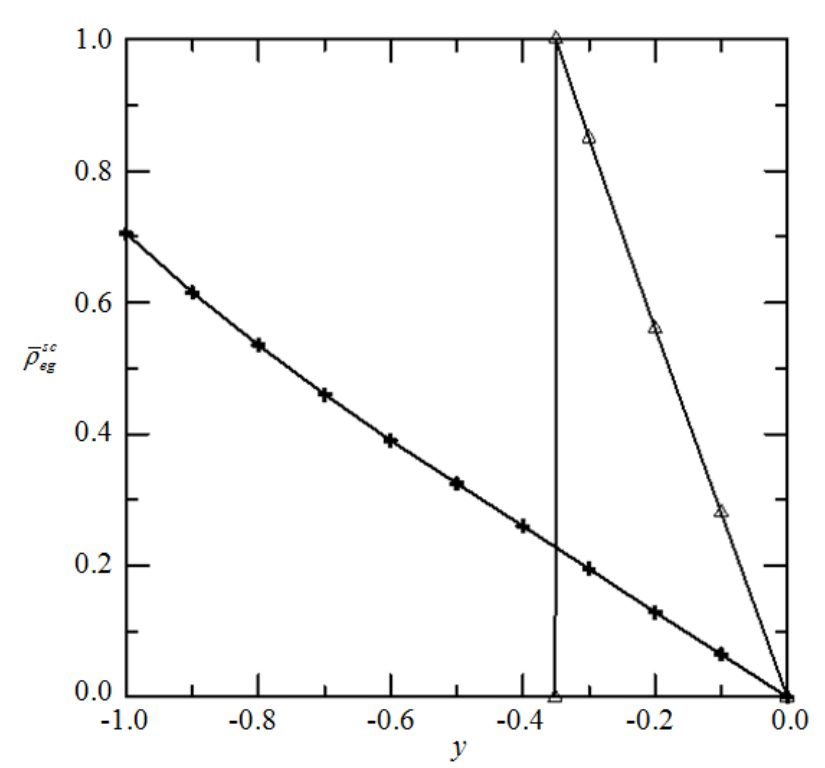

(b)

Fig. 3. (a) Semiconducting substrate: EG reduced DOS dependence on the dimensionless energy for $\approx 0.37,=2$ and (a), $\square 1.5$ (circles), 0 (squares), 1.5 (rhombs); Energies corresponding to the substrate energy gap, (b) Semiconducting substrate: EG reduced DOS dependence on the dimensionless energy for $\approx 0.37,=2$ and $(b)=0$, (crosses), 2 (triangles). Energy domain corresponding to the substrate valence band is shown

For $\varepsilon_{c}=0$ we arrive at:

$I \approx-\frac{2 \alpha V_{\text {aleg }}^{2}}{\xi^{2}} \omega \ln \left|\frac{\omega^{2}-\omega_{*}^{2}}{\omega^{2}}\right|$

where, $\omega_{*}=\omega_{+}=-\omega_{-}$.
For the semiconducting substrate the adatom's occupation number $n_{a}$ for the zero temperature is given by Equation 9. However, it is more convenient to represent $n_{a}$ as the sum of three terms. The first term is the contribution of the substrate valence band: 


$$
n_{v}=\int_{-\infty}^{-E_{g} / 2} \rho_{a}(\omega) d \omega
$$

where, $\rho_{a}(\omega)$ is defined by Equation 8, $\Gamma_{a}(\omega)=\pi V_{a / e g}^{2} \rho_{\text {val }}$ (i.e., $\Gamma_{\mathrm{a}}(\omega)=$ const) and $\Lambda_{a}(\omega)$ is defined by Equation 24-27.

Underline, that we take the lower integration limit equal to $-\infty$, which is considerably simplified calculation. It will be more correctly to take into account the finite band widths for semiconductor $W$ (see, for example, (Davydov, 2014a)) and graphene $D$ and define the bottom energy of the EG DOS as the lower limit of integration. Using, however, semiconductor DOS given by Equation 18, we arrive at (28).

The second term in $n_{a}$, i.e., $n_{e g}$, arises from the energy gap $E_{g}$ domain in the case when the Fermi level corresponds to the subband (21) energy interval. Thus:

$n_{e g}=\vartheta\left(\varepsilon_{F}-\omega_{-}\right) \cdot \frac{2}{\xi^{2}} \int_{\omega_{-}}^{\varepsilon_{F}}|\bar{\Omega}| d \omega$

where, $\vartheta(z)$-Heaviside step function, which is equal to1 for $z>0$ and 0 for $z<0$.

The third term is the local state contribution $n_{1}$, defined as:

$n_{l}=\left|1-\frac{d \Lambda_{a}(\omega)}{d \omega}\right|_{\omega_{l}}^{-1}$

where, $\omega_{l}$ is the energy of the local state lying below the bottom of the EG DOS. Since we have shifted this bottom to $-\infty$, the local state contribution (30) is absent.

Estimate now value of $n_{v}$. Since $\Gamma_{a}(\omega)=$ const, $\rho_{a}(\omega)$ function has maximum at energy $\omega^{\prime}$, which is the root of equation:

$\omega-\varepsilon_{a}-\Lambda_{a}(\omega)=0$

Let us suppose that for the energies $\omega<-E_{g} / 2$ shift function $\Lambda_{a}(\omega)$ is small except the narrow interval near the upper edge of the semiconductor valence band ($\left.E_{g} / 2\right)$. Putting approximately $\omega_{a}^{\prime} \approx \varepsilon_{a}+\Lambda_{a}\left(\varepsilon_{a}\right)$, we get $\rho_{a}(\omega) \approx \pi^{-1} \Gamma_{a} /\left[\left(\omega-\omega^{\prime}\right)^{2}+\Gamma_{a}^{2}\right]$, which gives with the use of Equation 28 the following expression:

$n_{v} \approx \frac{1}{\pi} \operatorname{arcctg} \frac{\varepsilon_{a}+\Lambda_{a}\left(\varepsilon_{a}\right)+E_{g} / 2}{\pi V_{a / e g}^{2} \rho_{v a l}}$

Note, that for the $p$-type substrate, when the Fermi level lies just near the top of the valence band, $n_{v}=n_{a}$.
Now consider some particular cases. Let $\varepsilon_{\mathrm{a}}<<-$ $E_{g} / 2$. Then we obtain from Equation 32 that $n_{v} \sim 1$, which means that the charge transfer between adatoms and EG is absent. For $\left(\varepsilon_{a}+E_{g} / 2\right) \sim 0$ it follows from (32) that $n_{v} \sim 0$. The same result is taken place for $\varepsilon_{a}>>-$ $E_{g} / 2$. In this case we have the complete charge transfer from adatom to EG. For the simplest case $\varepsilon_{c}=$ $\varepsilon_{a}=0$ we have:

$n_{v} \approx \frac{1}{\pi} \operatorname{arcctg} \frac{E_{g}}{2 \pi V_{\text {aleg }}^{2} \rho_{\text {val }}}$

Coarsen the real situation, we can rewrite Equation 33 in the form of $n_{v} \approx \pi^{-1} \operatorname{arcctg}\left(V_{g / s c}^{2} / V_{\text {aleg }}^{2}\right)$. Thus, the increase of the adatom-EG coupling compare to graphene-substrate coupling the valence band contribution to the resulting adatom's occupation number $n_{a}$ increases but the charge transfer decreases. Now turn to the estimation of $n_{\text {eg }}$. If $\omega_{-}<\varepsilon_{F}<\omega_{+}$, then:

$n_{e g}=\frac{2}{\xi^{2}} \int_{\omega_{-}}^{\varepsilon_{F}}\left|\omega-\varepsilon_{c}-\Lambda_{s c}(\omega)\right| d \omega$

Putting $\omega_{c}^{\prime} \approx \varepsilon_{c}+\Lambda_{s c}\left(\varepsilon_{c}\right)$ for $\varepsilon_{F} \leq \omega_{c}^{\prime}$, we find $n_{e g} \approx 1-2 \varepsilon_{F}\left(\omega_{c}^{\prime}-\varepsilon_{F}\right) / \xi^{2}$, for $\omega_{c}^{\prime}<\varepsilon_{F} \leq \omega_{+} \quad$ we get $n_{e g} \approx 1+2 \varepsilon_{F}\left(\varepsilon_{F}-\omega_{c}^{\prime}\right) / \xi^{2}$ and for $\varepsilon_{F}>\omega_{+}$we obtain $n_{e g}=$ 2. The value of $\varepsilon_{F}$ is, of course, calculated from the electron number conservation. Here, however, we omit this procedure since we are only interested in the relative effects of graphene and substrate on the value of $n_{a}$.

\section{Conclusion}

In this study we have constructed the scheme, which gives us possibility to calculate the charge transfer in the system adatom-EG. Estimations which have been done here show that the electronic state of adatom is affected by both gra-phene and substrate. What coupling is prevailed depends on the particular adsorption system.

Here we have considered only the isolated adatom. It is not very complicate to generalize obtained results to the finite adatom's concentrations taking into account their interactions (see, for example, (Davydov, 2012)). Difficulties, however, concern with the practical absence of the corresponding experimental data while it should be good to have such an information in the volume of, say, adsorption on metals (Braun and Medvedev, 1989). In this situation it is may be possible for the semi-quantity estimations to use the adsorption on graphite data (Caragiu and Finberg, 2005; Davydov, 2010). 


\section{Acknowledgement}

This study was supported in part by the program of the Government of Russian Federation (grant 074-U01).

\section{Funding Information}

This work was supported in part by the budget of Russian Federation.

\section{Author's Contributions}

Davydov Sergei: Maid all calculations and participated in all results analysis; contributed to the writing of the manuscript.

Lebedev Alexander: Participated in all results analysis and contributed to the writing of the manuscript; designed the research plan and organized the study.

\section{Ethics}

This article is original and contains unpublished material. The corresponding author confirms that all of the other authors have read and approved the manuscript and no ethical issues involved.

\section{References}

Anderson, P.W., 1961. Localized magnetic states in metals. Phys. Rev., 124: 41-41. DOI: 10.1103/PhysRev.124.41

Braun, M.O.M. and V.K. Medvedev, 1989. Interaction between particles adsorbed on metal surfaces. Sov. Phys. Uspechi., 32: 328-348.

DOI: 10.1070/PU1989v032n04ABEH002700

Caragiu, M. and S. Finberg, 2005. Alkali metal adsorption on graphite: A review. J. Phys. Condens. Matter, DOI: 10.1088/0953-8984/17/35/R02

Castro Neto, A.H., F. Guinea, N.M.R. Peres, K.S. Novoselov and A.K. Geim, 2008. The electronic properties of graphene. Rev. Mod. Phys., 81: 109-109. DOI: 10.1103/RevModPhys.81.109

Cooper, D.R., B. D'Anjou, N. Ghattamaneni, B. Harack and M. Hilke et al., 2011. Experimental review of graphene. ISRN Condens. Matter Phys.

DOI: $10.5402 / 2012 / 501686$
Davydov, S.Y. and G.I. Sabirova, 2011. Model of adsorption on graphene. Phys. Solid State, 53: 654-664. DOI: 10.1134/S1063783411030061

Davydov, S.Y., 2010. Alkali metal adsorption on graphite: Calculation of a work function variation in the Anderson-Newns-Muscat model. Applied Surf. Sci., 257: 1506-1510.

DOI: 10.1016/j.apsusc.2010.08.085

Davydov, S.Y., 2011a. Energy of bonding an adsorbed atom with a single-layer graphene. Phys. Solid State, 53: 2545-2545. DOI: $10.1134 / \mathrm{S} 1063783411120055$

Davydov, S.Y., 2011b. Charge transfer in epitaxial graphene-metal substrate system. Tech. Phys. Lett., 37: 476-477. DOI: 10.1134/S1063785011050191

Davydov, S.Y., 2012. Concentration dependence of the charge of atoms adsorbed on single-sheet graphene. Tech. Phys. Lett., 38: 175-177. DOI: $10.1134 / \mathrm{S} 1063785012020216$

Davydov, S.Y., 2013. On the specific features of the density of states of epitaxial graphene formed on metal and semiconductor substrates. Semiconductors, 47: 95-104. DOI: $10.1134 / \mathrm{S} 1063782613010090$

Davydov, S.Y., 2014a. On the charge transfer in the "single-sheet graphene-intercalated metal layer-SiC substrate" system. Phys. Solid State, 56: 412-419. DOI: $10.1134 /$ S1063783414020061

Davydov, S.Y., 2014b. Appearance conditions for a semiconducting-substrate-induced gap in the density of states in epitaxial graphene. Tech. Phys., 84: 624-627. DOI: $10.1134 / \mathrm{S} 1063784214040082$

Haas, J., W.A. de Heer, E.H. Conrad, 2008. The growth and morphology of epitaxial multilayer graphene. J. Phys. Condens. Matter, 20: 323202-323202. DOI: $10.1088 / 0953-8984 / 20 / 32 / 323202$

Wu, Y.H., T. Yu and Z.X. Shen, 2010. Two-dimensional carbon nanostructures: Fundamental properties, synthesis, characterization and potential applications. J. Appl. Phys., 108: 071301-071301. DOI: $10.1063 / 1.3460809$ 